\title{
A Novel Ebola Virus Spreading Mode
}

\author{
Yaqun $\mathrm{HUANG}^{1, \mathrm{a}}$, Haiyan $\mathrm{Ll}^{2, \mathrm{~b}}$, Yiming $\mathrm{CAO}^{3, \mathrm{c}}$, Ao Wang ${ }^{4, \mathrm{~d}}$, Pang $\mathrm{YANG}^{5, \mathrm{e}}$, \\ Xiaobing $\mathrm{ZHOU}^{6, \mathrm{f}}$ \\ ${ }^{1}$ School of Information Science and Engineering, Yunnan University, Kunming, 650091,China \\ ${ }^{2}$ School of Information Science and Engineering, Yunnan University, Kunming, 650091,China \\ ${ }^{3}$ School of Information Science and Engineering, Yunnan University, Kunming, 650091,China \\ ${ }^{4}$ School of Information Science and Engineering, Yunnan University, Kunming, 650091,China \\ ${ }^{5}$ School of Information Science and Engineering, Yunnan University, Kunming, 650091,China \\ ${ }^{6}$ School of Information Science and Engineering, Yunnan University, Kunming, 650091,China

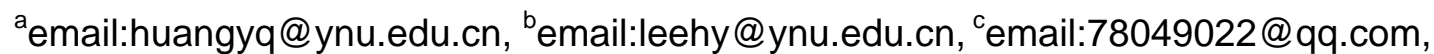 \\ demail:1248021774@qq.com, ${ }^{\text {eemail:1248021774@qq.com, }{ }^{f} e m a i l: z h o u x b @ y n u . e d u . c n ~}$
}

Keywords: S-E-I-R (Susceptible-Exposed-Infectious-Removed) model; Spatial network model of multi-population parameters; Staff travel process parameters

\begin{abstract}
A novel Ebola virus spreading model is proposed, choosing the mixed format of random transmission model to describe the population node transmission of infectious diseases within the process, using the deterministic S-E-I-R model to depict the internal mechanism of transmission of infectious diseases including infection, latent, and restore the four dynamic processing too,then putting the Gaussian white noise disturbance in the model and the transmission dynamics model which based on the individual, at last getting the evolution of the Ebola virus infection system behavior. According to the related algorithm to estimate and through the actual cases of infectious diseases data fitting, getting the local transmission of infectious diseases model parameters and the transmission probability areas.
\end{abstract}

\section{Introduction}

For the question of the Ebola virus spreading [1], we choose the mixed format of random transmission model to describe the population node transmission of infectious diseases within the process. People in the affected areas are divided into four categories: susceptible people, lurker, infections and outer people. Deterministic S-E-I-R model depicts the internal mechanism of transmission of infectious diseases including infection, latent, and restore the four dynamic processing. In order to import the random factors into the local transmission model, we put the Gaussian white noise disturbance in the model. In order to overcome the problem that it unable to describe the progress of the spread of infectious diseases at the individual level, we use the transmission dynamics model which based on the individual. We get the evolution of the Ebola virus infection system behavior, and then get the S-E-I-R dynamics equation of the differential form. According to the related algorithm to estimate and through the actual cases of infectious diseases data fitting, we get the parameters of the space multi group network model parameters, staff travel process parameters, local transmission model parameters and the transmission probability areas.

\section{Ebola Virus Dynamics SEIR Model}

We are using the mixed format of random SEIR (Susceptible-Exposed-Infectious-Removed) [2] model to describe the population of infection transmission within the process. Since this model is our stochastic and deterministic SEIR model developed on the basis of the SEIR model, we first introduce the deterministic SEIR model. Region $i$ of the $j$-th population of personnel within the node $\boldsymbol{N}_{i, j}$, we 
will be divided into four categories (bin): susceptible, exposed, infectious and removed, using $S_{i, j}$, $E_{i, j}, I_{i, j}, R_{i, j}$ to represent, i.e., $N_{i, j}=S_{i, j}+E_{i, j}+I_{i, j}+R_{i, j}$. Here emigration, including being kept completely separate, have recovered and they have immunity and deceased patients. The dynamical equation of change between the individuals in each bin can be described as follows:

$$
\left\{\begin{array}{l}
\frac{d S_{i, j}}{d t}=-\beta_{i} \frac{I_{i, j}}{N_{i, j}} S_{i, j} \\
\frac{d E_{i, j}}{d t}=-\beta_{i} \frac{I_{i, j}}{N_{i, j}} S_{i, j}-\gamma_{i} E_{i, j} \\
\frac{d I_{i, j}}{d t}=-\gamma_{i} E_{i, j}-\varphi_{i} I_{i, j} \\
\frac{d R_{i, j}}{d t}=\varphi_{i} I_{i, j}
\end{array}\right.
$$

In the type, $\beta_{i}, \gamma_{i}$ and $\varphi_{i}$ represent the area $i$ of the effective contact rate, risk and the probability of emigration. Given the initial conditions, the above model will produce a set of deterministic propagation curves. We would like to (1) the first equation as an example in the description. Assume that people living with a contact number for each time step of the $k_{i}$ and a susceptibility to infection after contact with an infected person is infected with probability $p_{i, s e}$, then an infection each time step of the effective contact rate is

$\beta_{i}=k_{i} p_{i, s e}$. Because infected people only come into contact with susceptible people may cause new infection, therefore susceptible to infection within each time step for the number of $k_{i} p_{i, s e} S_{i, j} / N_{i, j}$, so $I_{i, j}$ can produce for the number of people living with HIV infection each time step $k_{i} p_{i, s e} S_{i, j} / N_{i, j}$, written form of differential equation, there occurs following:

$$
\frac{d S_{i, j}}{d t}=-k_{i} p_{i, s e} \frac{S_{i, j}}{N_{i, j}} I_{i, j}=-\beta_{i} \frac{I_{i, j}}{N_{i, j}} S_{i, j} .
$$

Then, we obtain the first equation of model (1).

Deterministic SEIR model depicts the internal mechanism for the spread of infectious diseases, including infection, latent, onset and recovery (removed) these four dynamics. However, the actual process is essentially a random process of infection transmission, and deterministic SEIR model and the random factor is not taken into account, the random disturbance has important implications for the initial stages of the spread of infectious diseases [2]. For example, in the deterministic SEIR model, as long as the basic reproductive number is $R_{0}>1$, with probability 1 the transmission of outbreaks and for random SEIR model, in the initial stages of the spread of infectious diseases, since infections were rare, even the basic reproduction number

$R_{0}>1$ propagation may soon become extinct. Random factors to be introduced into local propagation models, we put the Gaussian white noise disturbance [2] in the model (1). The random transmission models are given by:

$$
\left\{\begin{array}{l}
\frac{d S_{i, j}}{d t}=-\beta_{i} \frac{I_{i, j}}{N_{i, j}} S_{i, j}+\sqrt{\beta_{i} \frac{I_{i, j}}{N_{i, j}} S_{i, j}} \xi_{1}(t) \\
\frac{d E_{i, j}}{d t}=-\beta_{i} \frac{I_{i, j}}{N_{i, j}} S_{i, j}-\gamma_{i} E_{i, j}-\sqrt{\beta_{i} \frac{I_{i, j}}{N_{i, j}} S_{i, j}} \xi_{1}(t)+\sqrt{\gamma_{i} E_{i, j}} \xi_{2}(t) \\
\frac{d I_{i, j}}{d t}=-\gamma_{i} E_{i, j}-\varphi_{i} I_{i, j}-\sqrt{\gamma_{i} E_{i, j}} \xi_{2}(t)+\sqrt{\varphi_{i} I_{i, j}} \xi_{3}(t) \\
\frac{d R_{i, j}}{d t}=\varphi_{i} I_{i, j}-\sqrt{\varphi_{i} I_{i, j}} \xi_{3}(t)
\end{array}\right.
$$


In the type, $\xi_{i}(t)(i=1,2,3)$ of which are independent and identically distributed Gaussian white noise function, the expected value is 0 , i.e. $\left\langle\xi_{i}(t)>=0\right.$. Stochastic amplitude is proportional to $1 / \sqrt{N_{i, j}}$, so as $N_{i, j} \rightarrow \infty$, equations (3) would revert equations (1). Equations (3) is considered random factors in the transmission, but as it is a macroscopic model, which describes the propagation of infectious diseases at the individual level. In order to overcome this problem, in [2] a transmission dynamics model on the individual models is used as follows:

$$
\left\{\begin{array}{l}
S_{i, j}+I_{i, j} \rightarrow E_{i, j}+I_{i, j}\left(p_{i, s e}\right) \\
E_{i, j} \rightarrow I_{i, j}\left(p_{i, e i}\right) \\
I_{i, j} \rightarrow R_{i, j}\left(p_{i, i r}\right)
\end{array} .\right.
$$

In this model, we set the number of individuals contacted for the $k_{i}$. Where the first expression is in each time step, if a susceptible contact with an infected person, the probability $p_{i, s e}$ of susceptible people to become sleeper.The second reaction is in each time step, lurk with probability $p_{i, e i}$ become infected. The third expression is in each time step, infected with probability $p_{i, i r}$ is moved out. Suppose at $t$ moment, susceptible persons, lurk in the system and the number of people living with $S_{i, j}, E_{i, j}, I_{i, j}$, respectively for the probability $p\left(S_{i, j}, E_{i, j}, I_{i, j} ; t\right)$, then the master equation of $p\left(S_{i, j}\right.$, $\left.E_{i, j}, I_{i, j} ; t\right)[2]$ is described as follows:

$$
\begin{aligned}
\frac{\partial p\left(S_{i, j}, E_{i, j}, I_{i, j} ; t\right)}{\partial t}= & \frac{\beta_{i}}{N_{i, j}}\left(S_{i, j}+1\right) I_{i, j} p\left(S_{i, j}+1, E_{i, j}-1, I_{i, j} ; t\right) \\
& +\gamma_{i}\left(E_{i, j}+1\right) p\left(S_{i, j}, E_{i, j}+1, I_{i, j}-1 ; t\right) \\
& +\varphi_{i}\left(I_{i, j}+1\right) p\left(S_{i, j}, E_{i, j}, I_{i, j}+1 ; t\right) \\
& -\left(\frac{\beta_{i}}{N_{i, j}} S_{i, j} I_{i, j}+\gamma_{i} E_{i, j}+\varphi_{i} I_{i, j}\right) p\left(S_{i, j}, E_{i, j}, I_{i, j} ; t\right)
\end{aligned}
$$

In the type, $\beta_{i}=k_{i} p_{i, ~ s e}, \gamma_{i}=p_{i, e i}, \varphi_{i}=p_{i, i}$, given initial conditions $p\left(S_{i, j}, E_{i, j}, I_{i, j} ; t=\right.$ $\left.t_{0}\right)=\delta_{I_{i, j-I_{0}}} \delta_{S_{i, j} N_{i, j-I_{0}}}$, assuming that the initial number of infected people is $I_{0}$, then model (5) describes the evolution of the epidemic system behavior. According to the literature $[2,3,4]$, when $N_{i, j}>>1$, we can use model (3) to approximate model (5), that is to say, in this case, model (3) and model (5) are actually equivalent. Here we only use the first equation of model (4) to give a simple and intuitive analysis. Due to an easy sense who each time step of contact number for $k_{i}$, such each time step on has $k_{i} I_{i, j} / N_{i, j}$ a infected and of contact, the easy sense who so and was infection of probability for $1-\left(1-p_{i, s e}\right)^{\frac{k_{i} I_{i, j}}{N_{i, j}}} \approx 1-\left(1-\frac{k_{i} p_{i, s e} I_{i, j}}{N_{i, j}}\right)=\frac{k_{i} p_{i, s e} I_{i, j}}{N_{i, j}}$. So, for the system with the easy sense number of $S_{i, j}$, average each time step of freshmen infected number for $k_{i} p_{i, s e} S_{i, j} I_{i, j} / N_{i, j}$ [2]. Thus, we have the following equation:

$$
\Delta S_{i, j}=-k_{i} p_{i, s e} \frac{I_{i, j}}{N_{i, j}} S_{i, j}=-\beta_{i} \frac{I_{i, j}}{N_{i, j}} S_{i, j} .
$$

This is what the first equation of model (1) finites the difference scheme, which corresponds to the certainly part of the first equation of model (1) . Theoretically, using model (4) as local propagation model is undoubtedly the best, because this allows you to simulate the spread of infectious diseases at the individual level, but from a practical perspective, the simulation of the model efficiency is relatively low, especially when the number of individuals more time. In fact, in this model, we need to track infections and having to keep track of susceptible people, so we can model (3) the first equation instead of model (4) the first equation in and retain model (4) in the second and third equation. By 
adopting a hybrid format of the SEIR model, both at an individual level to simulate the spread of infectious diseases, but also ensures the high computing speed.

\section{Spatial network model of multi-population parameters}

This type of argument is to determine population structure within each city. For the models (1),(3) and (5) , population size of lower value and ceiling value respectively set for $N_{\min }, N_{\max }$, population size power law distribution index for $\eta=3.0$, regulation network degrees distribution and side long distribution of parameter respectively for $\alpha=1.0, \beta=2.6$, then the network of degrees distribution for $p(k) \sim k^{-3}$, side long distribution for $p(\Delta r) \sim \Delta r^{-1.6}$, population node of average connection degrees for $<k>=2 m=6$.

\section{Staff travel process parameters}

\section{Area $i$ contact rate $\boldsymbol{k}_{j}$}

Contact rate number represents the time that one patient per unit of time in contacts with others. If a susceptible after contact with an infected person has been infected with probability $p_{i, s e}$, then $\beta_{i}=k_{i}$ $p_{i, s e}$ is called the adequate contact rate. A region's contact rate reflects the frequency of contacts between people in the region, and the area's population density, related to many factors such as the level of economic development. In this model, we assume that exposure is proportional to population density, that is:

$$
k_{i}=k_{\min }+\frac{\rho_{i}-\rho_{\min }}{\rho_{\text {max }}-\rho_{\text {min }}}\left(k_{\max }-k_{\min }\right) .
$$

In the type, $\rho_{i}$ is the population density of the region $i, \rho_{\min }, \rho_{\max }$ is respectively the minimum and maximum values in the population density of the city, $k_{\min }, k_{\max }$ is an artificial setting of exposure, respectively the lower and upper values.

\section{Transmission probability $p_{i, s e}$ of the area $i$}

We consider the actual process of infection transmission is divided into two phases: free dissemination phase and controlled dissemination phase. In free spread stage, infectious diseases also in low sent period, outbreak does not obviously, people on infectious diseases of prevention consciousness compared weak, at infectious diseases of infection probability can considered constants, that $p_{i, s e}=p_{s e}=$ const. When infectious diseases by low sent period entered rose period, people of personal prevention consciousness strengthened, and other hand, government will implemented related of public health measures, as travel limit, and access test and isolation, makes infectious diseases of infection probability with time gradually variable small, we said this stage for by controlled spread process. Controlled dissemination phase, infection probability curve fitting cases can be obtained according to the monitoring data, and therefore its function depends on specific infectious diseases. The transmission probability curve equation for exponential decay functions is as follows:

$$
p_{i, s e}(t)=p_{s e, \min }+p_{s e} \exp \left(-\frac{t-t_{\text {free }}}{\tau}\right)
$$

In the type, $t_{\text {free }}$ stands for infectious disease free propagation time, require $t>t_{\text {free }}$; $\tau$ is the characterization of probability of infection characteristic time scales of the decay rate; $p_{s e \text {, min }}$ is the limit of the minimum transmission probability.

\section{Conclusion}

In order to effectively control the spreading of Ebola, we build up a model to describe how the disease spread. And we use the four type people to be the crucial element in the model. From the model we have built, we can obtain the evolution of the Ebola virus infection system behavior. 
What's more, based on the Cases of infectious diseases in the epidemic area, we also get the local transmission of infectious diseases model parameters and the transmission probability areas.

\section{Acknowledgement}

The authors sincerely thank the reviewers and editors for their helpful comments and suggestions. This research was financially supported by the Natural Science Foundation of China (Project No. 11026225 and 61463050), and the Scientific Research Fund of Yunnan Education Department (Project No. 2014Y003).

\section{References}

[1] World Health Organization Ebola on http : // apps .who . int / ebola /en/ ebola -situation -report/ situation -reports / ebola-situation-report-4- february-2015.

[2] Shunjiang Ni. Research on Modeling of Infectious Disease Spreading Based on Complex Network Theory[D].Beijing: Tsinghua University,2009.

[3] Xianmin Meng, Dong Ping, Hongzhou Lu.Progress in the treatment of and new drug research on Ebola virus disease [J].Shanghai Medical \& Pharmaceutical Journal,2014(21)1-5.

[4] Qiuyue Gao, Luping Xiao, Haiyan Li, Xianping Fang.Ebola virus and immunological research progress [J].BIOLOGY TEACHING,2009:34(7):7-9.

[5] Qiyuan Jiang, Jinxing Xie,Ye Jun.Mathematical Model[M].Higher Education Press,2003. 